\title{
Variability Estimates for Yield Determining Characters in Common Bean (Phaseolus vulgaris L.)
}

\author{
Nancy Gupta ${ }^{1 *}$, Sajad Majeed Zargar ${ }^{2}$, R.K. Salgotra ${ }^{1}$, Manish Kumar Sharma ${ }^{1}$, \\ S.K. Gupta ${ }^{1}$ and G.K. Rai ${ }^{1}$ \\ ${ }^{1}$ Sher-e-Kashmi University of Agricultural Sciences and Technology of Jammu, \\ Chatha, Jammu, J\&K, 180009, India \\ ${ }^{2}$ Sher-e-Kashmi University of Agricultural Sciences \& Technology of Srinagar, Shalimar, \\ Srinagar, J\&K, 190025, India \\ *Corresponding author
}

\section{A B S T R A C T}

\section{Keywords}

Common bean, Morphological characters, Diversity

\section{Article Info}

Accepted:

04 July 2019

Available Online:

10 August 2019
The study herein determines the available variability among the unexplored common bean germplasm based on morphological parameters. The collection of one hundred and thirty five genotypes was done and the experimental data for nine phenotypic yield attributing traits was recorded in the experimental field of SKUAST Jammu, Main Campus, Chatha for the two years. The field experiment was laid out in Randomized Block Design and the observations were recorded from six randomly selected tagged plants. The analysis of variance of pooled data depicted remarkable variability among the genotypes for all the characters studied. The observations showed that the genotypes namely K12 (Bandipora) were early maturing, N3 (Nishat) had the longest pod, R3 (Rajouri) exhibit the largest pod width, MT6 (Marmat) had the maximum value of seed weight and that the genotypes namely P3 (Poonch), KS6 (Shopian), VLR125 (Almora, Uttarakhand) showed the highest yield. Comparatively higher values for seeds per plant and pods per plant parameters were noticed in the genotypes belonging to Almora (VLR-125), Poonch (P11), Shopian (KS1 and KS6), Marmat (MT5) and Uganda (UG5, UG6, UG9 and UG11) region. Further, the pods per plant, seeds per plant, 100 seed weight and seed yield per plant traits depicted higher estimates for PCV, GCV and genetic advance presenting great scope for accelerating the crop improvement. Based on these preliminary results, the elite diverse genotypes can be selected for future breeding programs thereby enhancing yield in common bean

\section{Introduction}

Common bean (Phaseolus vulgaris L.) is one of the most important widely cultivated species in Fabaceae (Leguminosae) family with 11 pairs of chromosomes $(2 n=2 x=22)$ and $473 \mathrm{Mb}$ genome size (Schmutz et al., 2014). The crop is predominantly selfpollinated, annual herbaceous, cultivated in tropical, semitropical and temperate regions of the world (Liebenberg, 2000). The two major gene pools of common bean (Mesoamerican 
and Andean) have been identified on account of various morphological, biochemical and molecular diversity studies (Cortes, 2013). Common bean genotypes of Andean gene pool are large-seeded whereas Middle American gene pool is characterized by medium and small size seeds. Within the Andean gene pool, the evolutionary races, Chile, Nueva, Granada and Peru have been identified whereas three evolutionary races, Durango, Jalisco (medium-seeded climber) and Mesoamerica (small seeded) have been characterized within the Middle American gene pool (Singh et al., 2000). The Middle American gene pool extends from Mexico through Central America and into Colombia and Venezuela, whereas the Andean gene pool is found in Peru, Chile, Bolivia and Argentina (Mamidi et al., 2011).

Phaseolus vulgaris commonly known as French bean, kidney bean, haricot bean, snap bean and navy bean belongs to Mesoamerican gene pool with Central and South America as major centers of origin (Wortmann et al., 2006). Due to its high nutritional value and potential to serve various pharmaceutical purposes, common bean is considered to be the most cultivated species among all the genotypes. Common bean accounts for more than 85 percent of the total world production of all cultivated Phaseolus species (Singh, 1999).

Out of the 70 Phaseolus species from Central and North America, only five (Phaseolus vulgaris: common bean; Phaseolus coccineus: runner bean; Phaseolus dumosus: year bean; Phaseolus acutifolius: tepary bean; Phaseolus lunatus: lima bean) are commonly domesticated (Freytag and Debouck, 2002; Gepts et al., 2008). P. dumosus and $P$. coccineus are more closely related to $P$. vulgaris that are partially intercrossable whereas P. lunatus and P. acutifolius are more distantly related (Belluci et al., 2014).
Common bean serves as the primary source of dietary proteins in developing countries in Latin America, Africa, and Asia (Messina, 1999). Being a rich source of quality proteins (20-25\%) containing amino acids such as methionine and lysine that is deficient in cereal seed (Bressani, 1983) and high amounts of energy sources such as carbohydrates, fibres, minerals ( $\mathrm{Fe}, \mathrm{Zn}, \mathrm{Ca}, \mathrm{Mg}, \mathrm{P}, \mathrm{K}, \mathrm{Na}, \mathrm{Mn}$ and Se), vitamins (Biotin and Vitamin A, B6), the crop is called as "poor man's meat" (WMO, 1992). They are also low in sodium and are gluten free (Agriculture and Agri-food Canada, 2009). It also contains a group of phenolic compounds such as flavonoids and bioflavonoids that play a crucial role in various anti-microbial defence mechanisms in plants. Besides this, it has proved its medicinal potential due to its anti-cancerous, antidepressant, anti-leukaemia, cardio-protective, estrogenic, hepatoprotective, chemopreventive, anti-diabetic, diauretic, diaphoretic, emmenagogue, fungicidal, hypoglycaemic, antiviral and antipyretic properties (Blumenthal et al., 1998; Rafi and Vastano, 2002).

Globally, 23 million hectares of common bean is grown worldwide (http://www.cgiar.org/ our-research/crop-factsheets/beans). Latin America is considered to be the largest producer of common bean followed by Brazil (Gepts et al., 2008). Among various developing countries, India ranks first in dry bean production whereas fourth in green bean production (FAO database, 2015). The average productivity in developed countries $(1,944 \mathrm{~kg} / \mathrm{ha})$ far exceeds to that of developing countries $(1,035 \mathrm{~kg} / \mathrm{ha})$ making the situation more drastic in least developed areas that mainly depend on beans as their primary dietary source (Gepts et al., 2008). An exponential increase in population size and current trends in bean consumption requires higher productivity and also the demand for $P$. vulgaris can be expected to increase (CIAT 
report 2001, http://webapp.ciat.cgiar.org/ ciatinfocus/beans.htm; Akibode and Maredia 2011). Palomina et al., (2012) reported the need for 30 percent increase in production of common bean by the year 2050. Hence, the continuous rise in population size and globalization presents challenges to the researchers for developing high yielding varieties with good agronomic merit.

In India, the crop is cultivated in various regions including Jammu and Kashmir, Himachal Pradesh, Uttarakhand, Uttar Pradesh, Maharashtra, Karnataka and Andhra Pradesh. It is a major commercial grain legume in the North-Western Himalayan region of India and is known for its high quality and flavor. A vast diversity exists in plant type, grain morphology and in agroecological adaptation of land races cultivated in this region, which is near the secondary centre of origin (Western China) of the species (Singh, 2000). However, very little efforts have been made to explore the germplasm/landraces from this region. On that account, we attempted to estimate the genetic variability among the germplasm by morphological evaluation of genotypes which forms the basis for improvement of any crop.

\section{Materials and Methods}

The germplasm taken under consideration for the present study comprised of one hundred and thirty five genotypes collected mainly from different regions of Jammu and Kashmir with one genotype procured from Almora, Uttarakhand. The details of the genotypes along with their source are presented in the table 1. The evaluation of collected germplasm was conducted for the two years viz. 2014 and 2015 at the experimental field of School of Biotechnology, SKUAST-Jammu (situated at $32^{\circ} 40^{\prime} \mathrm{N}$ latitude and $74^{\circ} 58^{\prime} \mathrm{E}$ longitude and has an altitude of $332 \mathrm{~m}$ above the mean sea level). The experimental material was grown in randomized block design (RBD) with two replications with row length of $2.0 \mathrm{~m}$ and spacing of $0.5 \mathrm{~m}$ (Figure 1 ).

Data were recorded for various yield attributing traits namely, days to bud initiation, days to flowering, days to fruiting, pod length, pod width, total number of pods per plant, total number of seeds per plant, 100 seed weight and seed yield per plant. The number of days taken after sowing for the plant to form buds, flowers and the first pod set were recorded as days to bud initiation, days to flowering and days to fruiting respectively. Pod length from six randomly selected pods of each tagged plant was measured from base of the pod to the tip of pod (leaving the stalk) using thread and scale and the observations were averaged for each tagged plant. The average mean of values of each tagged plants was calculated. The same pods used for recording length were also used for measuring the width. The width was measured from the middle portion of the pod and the averaged width was worked out. The number of pods from each picking of every tagged plant was recorded and the average of total number of pods per plant was calculated. The number of seeds from three tagged plants in each replication were counted and averaged. The seeds were harvested and the total weight of 100 seeds of each genotype was measured and recorded. The seeds were harvested and the weight of seeds of each tagged plant in a block was measured in grams and averaged to get the seed yield per plant.

Observations were recorded from three tagged plants of each block in both the replications and their average mean were worked out for statistical analysis. The pooled analysis of variance for nine traits was performed and analyzed where ' $F$ ' values depicted significant differences among the genotypes for all the characters. The genotypic and phenotypic coefficients of variability were calculated as 
per the method suggested by Burton and De Vane, (1953). Heritability in broad sense was calculated as per formulae given by Allard, (1960) and the genetic advance expressed as percent of population mean was calculated by the formula suggested by Johnson et al., (1955).

\section{Results and Discussion}

The performance of genotypes showed wide variation based on the analysis of variance which showed highly significant differences among the genotypes for all the recorded traits (Table 2). The mean performance for days to flowering varied from 51.25 days in K12 to 69.5 days in R7 and KS5 with overall general mean of 59.88. The data obtained for days to fruiting showed that genotype K12 had early pod set and genotypes R7 and KS5 took maximum days to bear fruit. The mean values for this trait obtained between 57.75 to 74.5 days with an average of 64.9 days. Pod length in collected common bean germplasm showed a wide variation ranging between $6.28 \mathrm{~cm}$ and $17.4 \mathrm{~cm}$ with an average of $11.4 \mathrm{~cm}$ size. The shortest pod was recorded for P10 genotype and longest pod seen for N3 genotype. Further, genotype B20 showed the minimum pod width of $0.63 \mathrm{~cm}$ whereas genotype R3 showed the highest value for pod width of $1.91 \mathrm{~cm}$ with an average mean of $1.17 \mathrm{~cm}$ pod width. Pods per plant varied from 5.64 to 21.67 with minimum found in KS5 and maximum obtained for VLR125 with an average of 11.09 pods per plant recorded. Genotype P19 showed minimum seeds per plant while maximum was recorded for genotype VLR125. The values varied from 22.1 to 110.98 with an average of 50.42. A huge variation was found for 100 seed weight trait where the values ranged between $11 \mathrm{~g}$ in case of KS9 to $100.2 \mathrm{~g}$ in case of MT6 with an average of $25.6 \mathrm{~g}$. The average seed yield per plant was $12.6 \mathrm{~g}$ ranging between $2.83 \mathrm{~g}$ and $45.83 \mathrm{~g}$ with minimum obtained for UD6 and maximum for KS6.

Table.1 List of common bean genotypes used in the present study along with their respective place of collection

\begin{tabular}{|c|c|c|c|}
\hline $\begin{array}{c}\text { S. } \\
\text { No. }\end{array}$ & Genotypes & $\begin{array}{c}\text { Number of } \\
\text { Genotypes }\end{array}$ & Source \\
\hline $\mathbf{1}$ & P1 to P19 & 19 & Poonch \\
\hline $\mathbf{2}$ & R1 to R10 & 10 & Rajouri \\
\hline $\mathbf{3}$ & KS1 to KS11 & 11 & Shopian \\
\hline $\mathbf{4}$ & K12, K14, K15, K16, KS18 & 5 & Bandipora \\
\hline $\mathbf{5}$ & K13, KS17, KS19 & 3 & Baramulla \\
\hline $\mathbf{6}$ & K20 & 1 & Qazigand \\
\hline $\mathbf{7}$ & B1 to B20 & 20 & Baderwah \\
\hline $\mathbf{8}$ & MT1 to MT7 & 7 & Marmat \\
\hline $\mathbf{9}$ & MR1 to MR2 & 2 & Marwah \\
\hline $\mathbf{1 0}$ & DE1 to DE4 & 4 & Dessa \\
\hline $\mathbf{1 1}$ & UG1 to UG14 & 14 & Ugada \\
\hline $\mathbf{1 2}$ & N1 to N7, N9 to N11, N13 to N17 & 6 & Nishat \\
\hline $\mathbf{1 3}$ & UD1 to UD6 & 17 & Northampur \\
\hline $\mathbf{1 4}$ & KD1 to KD11, KD13 to KD18 & & Kashmir \\
\hline & & 1 & Almora \\
\hline $\mathbf{1 5}$ & VLR-125 & 135 & \\
\hline Total & & & \\
\hline
\end{tabular}


Table.2 Mean performance of various characters under study in common bean (Phaseolus vulgaris L.)

\begin{tabular}{|c|c|c|c|c|c|c|c|c|c|}
\hline Genotypes & $\begin{array}{l}\text { Days to bud } \\
\text { initiation }\end{array}$ & $\begin{array}{l}\text { Days to } \\
\text { flowering }\end{array}$ & $\begin{array}{l}\text { Days to } \\
\text { fruiting }\end{array}$ & $\begin{array}{l}\text { Pod length } \\
\text { (cm) }\end{array}$ & $\begin{array}{l}\text { Pod width } \\
\text { (cm) }\end{array}$ & Pods/plant & Seeds/plant & $\begin{array}{l}100 \text { seed } \\
\text { weight }(g)\end{array}$ & $\begin{array}{l}\text { Seed } \\
\text { yield/plant }\end{array}$ \\
\hline P1 & 53.75 & 58.00 & 63.75 & 10.01 & 1.28 & 11.49 & 46.50 & 21.41 & 9.70 \\
\hline P2 & 51.75 & 56.50 & 61.50 & 9.35 & 0.99 & 19.33 & 87.60 & 20.28 & 17.82 \\
\hline P3 & 61.75 & 66.50 & 71.50 & 13.28 & 1.39 & 16.14 & 69.70 & 41.70 & 29.02 \\
\hline P4 & 53.75 & 57.50 & 63.00 & 11.51 & 1.13 & 7.26 & 32.53 & 35.63 & 11.55 \\
\hline P5 & 55.75 & 60.25 & 65.00 & 10.03 & 1.18 & 9.72 & 39.75 & 22.27 & 8.81 \\
\hline P6 & 57.25 & 61.25 & 66.00 & 12.23 & 1.19 & 12.89 & 40.55 & 23.38 & 9.62 \\
\hline P7 & 53.75 & 60.50 & 66.25 & 9.82 & 0.69 & 11.02 & 54.02 & 15.63 & 8.44 \\
\hline P8 & 53.25 & 56.75 & 61.25 & 12.24 & 1.16 & 8.50 & 35.20 & 30.92 & 10.85 \\
\hline P9 & 54.25 & 60.50 & 66.50 & 7.88 & 1.04 & 15.62 & 53.88 & 19.44 & 10.01 \\
\hline P10 & 58.25 & 62.25 & 67.75 & 6.28 & 1.09 & 10.94 & 50.03 & 11.60 & 5.83 \\
\hline P11 & 52.75 & 57.50 & 62.75 & 10.45 & 1.21 & 19.56 & 102.24 & 21.23 & 22.27 \\
\hline P12 & 51.25 & 55.00 & 62.50 & 10.85 & 0.99 & 11.53 & 46.03 & 19.63 & 9.04 \\
\hline P13 & 54.25 & 60.75 & 66.00 & 12.07 & 1.13 & 6.27 & 23.60 & 15.62 & 3.85 \\
\hline P14 & 61.75 & 66.00 & 70.50 & 10.93 & 1.28 & 8.14 & 34.20 & 12.58 & 4.35 \\
\hline P15 & 56.25 & 65.25 & 69.50 & 11.61 & 0.80 & 9.19 & 37.21 & 13.37 & 4.87 \\
\hline P16 & 59.75 & 65.00 & 69.25 & 11.20 & 1.01 & 10.29 & 45.25 & 25.62 & 11.64 \\
\hline P17 & 57.75 & 63.25 & 71.75 & 6.78 & 1.18 & 7.19 & 28.20 & 16.68 & 5.37 \\
\hline P18 & 57.25 & 60.00 & 64.25 & 12.15 & 0.84 & 6.89 & 26.40 & 20.76 & 5.52 \\
\hline P19 & 61.25 & 64.00 & 68.25 & 9.49 & 1.17 & 6.17 & 22.10 & 16.75 & 3.81 \\
\hline R1 & 55.75 & 59.00 & 63.25 & 13.58 & 1.05 & 7.81 & 33.60 & 27.04 & 9.08 \\
\hline $\mathbf{R 2}$ & 60.00 & 65.00 & 71.00 & 12.18 & 1.31 & 8.44 & 40.33 & 34.96 & 13.25 \\
\hline $\mathbf{R 3}$ & 55.25 & 59.50 & 63.75 & 11.10 & 1.91 & 7.62 & 29.10 & 39.17 & 11.64 \\
\hline R4 & 55.00 & 59.00 & 64.50 & 10.38 & 1.26 & 8.61 & 38.90 & 20.70 & 8.31 \\
\hline R5 & 57.50 & 61.25 & 65.75 & 9.80 & 1.16 & 20.75 & 91.60 & 20.02 & 18.56 \\
\hline R6 & 60.75 & 66.00 & 69.75 & 13.73 & 1.47 & 10.18 & 39.90 & 26.64 & 10.77 \\
\hline R7 & 65.25 & 69.50 & 74.50 & 12.10 & 1.10 & 15.25 & 71.30 & 27.58 & 19.99 \\
\hline R8 & 54.25 & 59.50 & 64.00 & 12.20 & 1.49 & 10.67 & 45.50 & 42.48 & 19.45 \\
\hline R9 & 54.75 & 59.25 & 64.75 & 9.55 & 1.15 & 15.68 & 72.02 & 20.51 & 14.85 \\
\hline R10 & 64.75 & 69.25 & 74.00 & 9.34 & 0.94 & 6.44 & 23.10 & 14.53 & 3.33 \\
\hline KS1 & 57.75 & 62.25 & 67.50 & 10.05 & 1.11 & 20.32 & 105.89 & 17.08 & 18.73 \\
\hline KS2 & 60.75 & 64.00 & 68.75 & 11.05 & 1.09 & 17.58 & 84.80 & 20.35 & 17.86 \\
\hline KS3 & 63.25 & 68.50 & 73.00 & 11.33 & 1.00 & 8.39 & 34.20 & 31.17 & 13.23 \\
\hline KS4 & 55.25 & 59.50 & 63.75 & 12.15 & 1.66 & 8.61 & 36.90 & 16.55 & 6.26 \\
\hline KS5 & 62.75 & 69.50 & 74.50 & 13.95 & 1.30 & 5.64 & 52.84 & 11.92 & 6.27 \\
\hline KS6 & 52.75 & 58.25 & 63.25 & 17.08 & 1.05 & 17.95 & 99.23 & 43.87 & 45.83 \\
\hline KS7 & 60.25 & 64.25 & 69.00 & 11.40 & 1.19 & 8.39 & 33.90 & 20.64 & 6.83 \\
\hline KS8 & 59.75 & 64.50 & 69.00 & 10.58 & 0.86 & 6.61 & 27.60 & 18.68 & 5.28 \\
\hline KS9 & 60.25 & 64.00 & 68.25 & 9.05 & 0.95 & 9.29 & 39.99 & 11.00 & 4.27 \\
\hline KS10 & 51.25 & 55.50 & 59.75 & 10.30 & 1.29 & 11.83 & 50.25 & 21.97 & 10.88 \\
\hline KS11 & 52.00 & 57.00 & 61.00 & 10.60 & 1.21 & 8.64 & 35.50 & 27.40 & 9.82 \\
\hline K12 & 47.75 & 51.25 & 57.75 & 12.10 & 1.51 & 6.82 & 26.40 & 77.18 & 20.88 \\
\hline K13 & 51.50 & 57.75 & 63.75 & 13.10 & 1.54 & 8.82 & 36.90 & 38.08 & 14.42 \\
\hline K14 & 51.75 & 56.00 & 62.25 & 11.40 & 1.44 & 9.20 & 40.20 & 34.88 & 13.77 \\
\hline K15 & 53.75 & 58.75 & 63.25 & 12.03 & 1.49 & 9.12 & 40.99 & 40.88 & 16.04 \\
\hline K16 & 62.00 & 66.50 & 71.00 & 10.35 & 0.94 & 10.19 & 42.12 & 20.14 & 8.62 \\
\hline K17 & 53.50 & 57.75 & 62.50 & 9.75 & 0.86 & 6.32 & 22.70 & 15.70 & 3.69 \\
\hline K18 & 55.00 & 59.25 & 64.25 & 10.15 & 1.21 & 6.53 & 25.10 & 18.85 & 4.72 \\
\hline K19 & 53.75 & 58.25 & 62.75 & 9.65 & 0.94 & 6.39 & 23.11 & 17.98 & 4.14 \\
\hline K20 & 60.25 & 65.25 & 68.75 & 10.78 & 0.98 & 9.13 & 38.90 & 13.26 & 4.96 \\
\hline B1 & 53.75 & 59.50 & 65.00 & 9.70 & 1.11 & 16.90 & 79.82 & 20.73 & 16.37 \\
\hline B2 & 52.75 & 59.75 & 64.75 & 8.78 & 0.89 & 9.97 & 42.75 & 14.71 & 6.59 \\
\hline B3 & 52.75 & 56.00 & 60.25 & 10.63 & 0.89 & 15.77 & 65.72 & 18.17 & 11.64 \\
\hline B4 & 56.75 & 62.00 & 67.50 & 13.10 & 1.11 & 5.83 & 18.77 & 59.25 & 10.28 \\
\hline B5 & 53.75 & 59.25 & 65.00 & 13.83 & 1.15 & 10.08 & 33.38 & 30.08 & 10.01 \\
\hline B6 & 54.25 & 59.25 & 63.50 & 14.13 & 1.23 & 7.17 & 26.95 & 46.03 & 12.37 \\
\hline B7 & 55.25 & 60.00 & 66.25 & 10.05 & 1.01 & 13.16 & 62.33 & 19.43 & 12.27 \\
\hline B8 & 54.00 & 57.50 & 62.50 & 9.05 & 0.91 & 7.71 & 28.73 & 12.03 & 3.48 \\
\hline
\end{tabular}


Int.J.Curr.Microbiol.App.Sci (2019) 8(8): 47-57

\begin{tabular}{|c|c|c|c|c|c|c|c|c|c|}
\hline B9 & 52.25 & 55.75 & 62.00 & 12.35 & 1.13 & 6.30 & 27.92 & 33.37 & 9.56 \\
\hline B10 & 52.25 & 55.75 & 61.50 & 13.63 & 1.58 & 11.02 & 51.23 & 43.36 & 22.99 \\
\hline B11 & 59.25 & 63.25 & 67.75 & 15.50 & 1.53 & 9.87 & 39.84 & 27.48 & 10.49 \\
\hline B12 & 54.25 & 58.50 & 63.50 & 11.60 & 1.04 & 14.45 & 58.50 & 15.74 & 9.51 \\
\hline B13 & 54.25 & 60.00 & 65.50 & 13.78 & 1.35 & 7.08 & 27.30 & 18.54 & 5.01 \\
\hline B14 & 55.75 & 59.75 & 65.00 & 11.13 & 1.98 & 7.54 & 28.70 & 27.02 & 7.95 \\
\hline B15 & 55.75 & 59.25 & 64.25 & 9.45 & 1.4 & 9.70 & 40.25 & 23.07 & 9.41 \\
\hline B16 & 55.25 & 59.75 & 64.75 & 11.53 & 1.19 & 18.25 & 85.30 & 24.13 & 20.69 \\
\hline B17 & 53.25 & 58.00 & 63.00 & 11.83 & 1.45 & 10.68 & 50.23 & 28.24 & 14.19 \\
\hline B18 & 54.75 & 58.25 & 63.50 & 10.40 & 1.03 & 17.11 & 79.81 & 15.14 & 12.22 \\
\hline B19 & 56.25 & 60.75 & 66.00 & 11.55 & 1.08 & 18.56 & 96.51 & 22.41 & 22.95 \\
\hline B20 & 54.75 & 59.50 & 64.75 & 10.18 & 0.63 & 7.52 & 34.91 & 21.18 & 7.22 \\
\hline MT1 & 54.75 & 59.00 & 64.50 & 10.00 & 0.69 & 9.00 & 40.81 & 29.55 & 11.67 \\
\hline MT2 & 56.25 & 60.25 & 66.50 & 12.25 & 1.45 & 10.84 & 49.50 & 26.70 & 13.14 \\
\hline MT3 & 60.25 & 64.00 & 70.00 & 9.83 & 1.35 & 15.85 & 67.06 & 16.27 & 10.55 \\
\hline MT4 & 57.75 & 62.00 & 67.00 & 13.60 & 1.50 & 14.35 & 67.90 & 21.85 & 14.33 \\
\hline MT5 & 58.75 & 62.75 & 68.00 & 10.38 & 0.93 & 18.23 & 102.36 & 14.25 & 15.94 \\
\hline MT6 & 54.75 & 58.25 & 63.25 & 15.43 & 1.31 & 4.50 & 13.82 & 100.2 & 13.27 \\
\hline MT7 & 54.75 & 60.50 & 66.00 & 11.35 & 1.21 & 9.12 & 37.56 & 27.09 & 9.99 \\
\hline MR1 & 51.75 & 56.00 & 61.25 & 9.95 & 0.99 & 10.93 & 46.65 & 24.49 & 11.21 \\
\hline MR2 & 53.25 & 58.00 & 63.25 & 8.80 & 1.24 & 15.90 & 72.60 & 27.50 & 19.91 \\
\hline DE1 & 54.00 & 60.25 & 65.25 & 14.25 & 1.18 & 9.65 & 39.18 & 35.81 & 13.73 \\
\hline DE2 & 56.00 & 60.00 & 65.25 & 12.35 & 1.03 & 10.33 & 37.61 & 19.30 & 7.10 \\
\hline DE3 & 59.00 & 62.75 & 67.75 & 9.38 & 0.93 & 10.66 & 46.05 & 18.07 & 8.28 \\
\hline DE4 & 56.50 & 60.75 & 65.75 & 10.33 & 1.40 & 8.47 & 35.90 & 28.48 & 10.12 \\
\hline UG1 & 56.75 & 61.25 & 67.00 & 9.03 & 0.95 & 17.11 & 83.56 & 11.30 & 9.45 \\
\hline UG2 & 54.75 & 59.75 & 65.50 & 10.35 & 1.21 & 13.29 & 56.61 & 17.83 & 10.06 \\
\hline UG3 & 55.00 & 60.00 & 65.25 & 10.75 & 0.96 & 13.49 & 63.73 & 22.78 & 14.34 \\
\hline UG4 & 53.75 & 58.25 & 63.75 & 14.20 & 1.55 & 10.95 & 62.99 & 28.08 & 17.56 \\
\hline UG5 & 60.25 & 65.00 & 69.50 & 10.30 & 1.21 & 19.98 & 105.24 & 18.80 & 20.99 \\
\hline UG6 & 55.75 & 60.50 & 64.75 & 10.18 & 0.91 & 18.56 & 101.54 & 19.94 & 20.40 \\
\hline UG7 & 58.25 & 62.75 & 68.25 & 11.98 & 1.24 & 9.46 & 45.23 & 26.20 & 11.72 \\
\hline UG8 & 55.25 & 59.25 & 64.00 & 12.38 & 1.16 & 7.15 & 33.12 & 77.45 & 25.38 \\
\hline UG9 & 54.00 & 58.50 & 64.00 & 15.28 & 1.46 & 20.64 & 107.92 & 22.91 & 25.63 \\
\hline UG10 & 56.25 & 60.25 & 64.75 & 10.58 & 1.15 & 9.23 & 40.93 & 22.03 & 9.13 \\
\hline UG11 & 54.00 & 61.25 & 66.25 & 14.50 & 1.78 & 19.56 & 102.56 & 23.31 & 24.78 \\
\hline UG12 & 59.75 & 63.00 & 67.75 & 10.10 & 1.10 & 9.76 & 39.75 & 20.61 & 6.12 \\
\hline UG13 & 53.25 & 58.50 & 64.25 & 8.30 & 1.61 & 16.99 & 82.12 & 17.09 & 13.92 \\
\hline UG14 & 58.50 & 62.75 & 66.50 & 11.18 & 1.40 & 12.28 & 53.12 & 23.10 & 12.09 \\
\hline N1 & 56.50 & 61.00 & 64.75 & 13.30 & 1.15 & 6.58 & 23.62 & 17.36 & 4.19 \\
\hline N2 & 51.25 & 54.50 & 58.75 & 11.38 & 1.11 & 11.04 & 41.10 & 23.16 & 8.95 \\
\hline N3 & 53.25 & 57.25 & 62.50 & 17.45 & 1.05 & 7.38 & 26.21 & 30.53 & 8.03 \\
\hline N4 & 60.50 & 65.00 & 69.00 & 11.38 & 1.17 & 9.42 & 41.23 & 14.07 & 5.85 \\
\hline N5 & 52.50 & 57.50 & 63.00 & 11.05 & 1.19 & 8.11 & 38.26 & 17.90 & 8.51 \\
\hline N6 & 53.25 & 58.25 & 63.00 & 12.98 & 1.29 & 6.76 & 28.73 & 34.84 & 9.99 \\
\hline N7 & 50.50 & 55.75 & 60.50 & 14.13 & 1.24 & 5.98 & 25.72 & 36.34 & 9.19 \\
\hline N9 & 51.75 & 55.25 & 60.75 & 11.23 & 1.19 & 8.74 & 37.91 & 36.88 & 14.08 \\
\hline N10 & 53.00 & 57.50 & 63.25 & 10.90 & 1.14 & 8.15 & 36.12 & 22.61 & 8.26 \\
\hline N11 & 53.00 & 57.50 & 62.75 & 12.60 & 1.00 & 8.54 & 38.82 & 30.13 & 11.65 \\
\hline N13 & 58.75 & 63.50 & 67.50 & 10.83 & 1.18 & 15.89 & 77.71 & 23.54 & 18.79 \\
\hline N14 & 53.25 & 58.00 & 64.25 & 10.35 & 0.79 & 8.99 & 38.21 & 21.20 & 8.07 \\
\hline N15 & 53.25 & 56.75 & 62.75 & 12.10 & 0.90 & 8.98 & 36.93 & 33.91 & 13.09 \\
\hline N16 & 52.50 & 57.50 & 62.75 & 12.65 & 0.83 & 7.92 & 31.11 & 34.80 & 10.77 \\
\hline N17 & 52.00 & 55.25 & 59.25 & 11.75 & 1.10 & 6.20 & 28.24 & 26.79 & 7.83 \\
\hline UD1 & 57.25 & 60.50 & 65.00 & 10.15 & 0.98 & 19.45 & 94.24 & 23.21 & 21.97 \\
\hline UD2 & 53.50 & 58.25 & 63.75 & 11.18 & 1.12 & 8.33 & 34.15 & 30.07 & 10.44 \\
\hline UD3 & 53.75 & 58.25 & 62.00 & 9.90 & 1.14 & 7.86 & 37.65 & 19.94 & 7.39 \\
\hline UD4 & 53.25 & 58.00 & 62.00 & 12.01 & 1.24 & 7.11 & 24.12 & 17.02 & 4.06 \\
\hline UD5 & 56.50 & 60.50 & 65.75 & 10.28 & 1.83 & 7.29 & 36.16 & 23.43 & 8.60 \\
\hline UD6 & 55.25 & 59.50 & 63.50 & 9.83 & 0.71 & 6.42 & 24.67 & 11.93 & 2.83 \\
\hline KD1 & 54.75 & 58.50 & 63.50 & 12.35 & 1.16 & 6.30 & 23.62 & 24.13 & 5.75 \\
\hline KD2 & 54.75 & 58.00 & 62.50 & 10.33 & 1.11 & 10.92 & 43.42 & 19.77 & 8.77 \\
\hline KD3 & 53.50 & 57.50 & 63.25 & 12.95 & 1.18 & 7.61 & 26.95 & 41.73 & 13.34 \\
\hline
\end{tabular}




\begin{tabular}{|c|c|c|c|c|c|c|c|c|c|}
\hline KD4 & 53.25 & 56.75 & 62.00 & 10.05 & 0.99 & 6.58 & 24.95 & 13.53 & 3.33 \\
\hline KD5 & 52.25 & 54.75 & 60.00 & 15.78 & 1.47 & 8.63 & 34.12 & 30.04 & 10.32 \\
\hline KD6 & 53.75 & 58.00 & 64.50 & 10.15 & 0.97 & 21.29 & 70.99 & 12.66 & 9.01 \\
\hline KD7 & 55.75 & 60.00 & 65.00 & 11.50 & 1.14 & 17.20 & 99.78 & 21.96 & 22.62 \\
\hline KD8 & 64.00 & 68.25 & 73.25 & 9.40 & 1.21 & 6.86 & 29.12 & 14.47 & 4.37 \\
\hline KD9 & 51.50 & 55.00 & 60.00 & 11.63 & 1.00 & 7.40 & 28.81 & 36.08 & 10.41 \\
\hline KD10 & 53.25 & 57.00 & 61.00 & 17.08 & 1.53 & 7.06 & 28.62 & 26.22 & 7.64 \\
\hline KD11 & 60.25 & 65.00 & 69.75 & 10.65 & 0.95 & 9.45 & 39.42 & 17.43 & 6.84 \\
\hline KD13 & 52.25 & 56.50 & 60.75 & 12.38 & 1.32 & 9.07 & 39.21 & 27.78 & 10.92 \\
\hline KD14 & 52.25 & 54.75 & 60.00 & 14.43 & 1.18 & 9.00 & 37.21 & 34.02 & 12.86 \\
\hline KD15 & 53.00 & 56.25 & 61.75 & 11.55 & 1.18 & 8.46 & 34.93 & 35.43 & 12.48 \\
\hline KD16 & 53.75 & 58.25 & 63.75 & 11.95 & 1.25 & 9.40 & 44.75 & 25.43 & 11.55 \\
\hline KD17 & 51.25 & 55.25 & 60.25 & 12.80 & 1.12 & 8.84 & 35.92 & 29.89 & 10.77 \\
\hline KD18 & 51.50 & 55.25 & 60.00 & 10.53 & 0.79 & 9.80 & 36.65 & 22.86 & 7.81 \\
\hline VLR125 & 49.00 & 54.25 & 59.00 & 13.25 & 1.33 & 21.56 & 110.98 & 31.15 & 29.12 \\
\hline SE m \pm & 0.43 & 0.53 & 0.72 & 0.38 & 0.06 & 1.32 & 6.15 & 1.38 & 1.47 \\
\hline CV & 1.09 & 1.25 & 1.56 & 4.68 & 6.97 & 17.18 & 18.20 & 5.41 & 17.77 \\
\hline CD & 1.19 & 1.48 & 2.00 & 1.06 & 0.16 & 3.59 & 17.21 & 3.86 & 4.11 \\
\hline
\end{tabular}

Table.3 Estimates of variability parameters for various characters in common bean

\begin{tabular}{|c|c|c|c|c|c|c|c|}
\hline \multirow[t]{2}{*}{ Characters } & \multirow[t]{2}{*}{$\begin{array}{c}\text { Mean } \pm \\
\text { SE }\end{array}$} & \multirow[t]{2}{*}{ Range } & \multicolumn{2}{|c|}{$\begin{array}{c}\text { Coefficient } \\
\text { of variation } \\
(\%)\end{array}$} & \multirow{2}{*}{$\begin{array}{c}\text { Heritability } \\
\% \\
\text { (Broad } \\
\text { sense) }\end{array}$} & \multirow[t]{2}{*}{$\begin{array}{c}\text { Genetic } \\
\text { advance }\end{array}$} & \multirow{2}{*}{$\begin{array}{c}\text { Genetic } \\
\text { advance } \\
\text { as \% of } \\
\text { mean }\end{array}$} \\
\hline & & & PCV & GCV & & & \\
\hline $\begin{array}{l}\text { Days to bud } \\
\text { initiation }\end{array}$ & $\begin{array}{l}55.43 \pm \\
0.43\end{array}$ & $47.75-65.25$ & 6.07 & 5.97 & 0.97 & 6.71 & 12.10 \\
\hline $\begin{array}{l}\text { Days to } 50 \% \\
\text { flowering }\end{array}$ & $\begin{array}{l}59.88 \pm \\
0.53\end{array}$ & $51.25-69.50$ & 5.90 & 5.76 & 0.96 & 6.95 & 11.60 \\
\hline $\begin{array}{l}\text { Days to } \\
\text { fruiting }\end{array}$ & $\begin{array}{l}64.93 \pm \\
0.72\end{array}$ & $57.75-74.50$ & 5.36 & 5.13 & 0.92 & 6.57 & 10.12 \\
\hline Pod length & $\begin{array}{l}11.43 \pm \\
0.38\end{array}$ & $6.28-17.40$ & 16.97 & 16.31 & 0.92 & 3.69 & 32.30 \\
\hline Pod width & $\begin{array}{l}1.17 \pm \\
0.06\end{array}$ & $0.63-1.91$ & 21.79 & 20.04 & 0.85 & 0.45 & 38.74 \\
\hline Pods/plant & $\begin{array}{l}10.85 \pm \\
1.32\end{array}$ & $5.64-21.56$ & 41.03 & 37.26 & 0.82 & 7.52 & 69.31 \\
\hline Seeds/plant & $\begin{array}{l}47.71 \pm \\
6.15\end{array}$ & $\begin{array}{l}22.10- \\
110.98\end{array}$ & 50.32 & 46.91 & 0.86 & 42.54 & 89.16 \\
\hline $\begin{array}{l}100 \text { seed } \\
\text { weight }\end{array}$ & $\begin{array}{l}25.56 \pm \\
2.74\end{array}$ & $\begin{array}{l}11.00- \\
100.20\end{array}$ & 49.08 & 48.80 & 0.99 & 25.58 & 100.09 \\
\hline $\begin{array}{l}\text { Seed } \\
\text { yield/plant }\end{array}$ & $\begin{array}{l}11.66 \pm \\
1.47\end{array}$ & $2.83-29.12$ & 56.64 & 53.78 & 0.90 & 12.13 & 103.40 \\
\hline
\end{tabular}



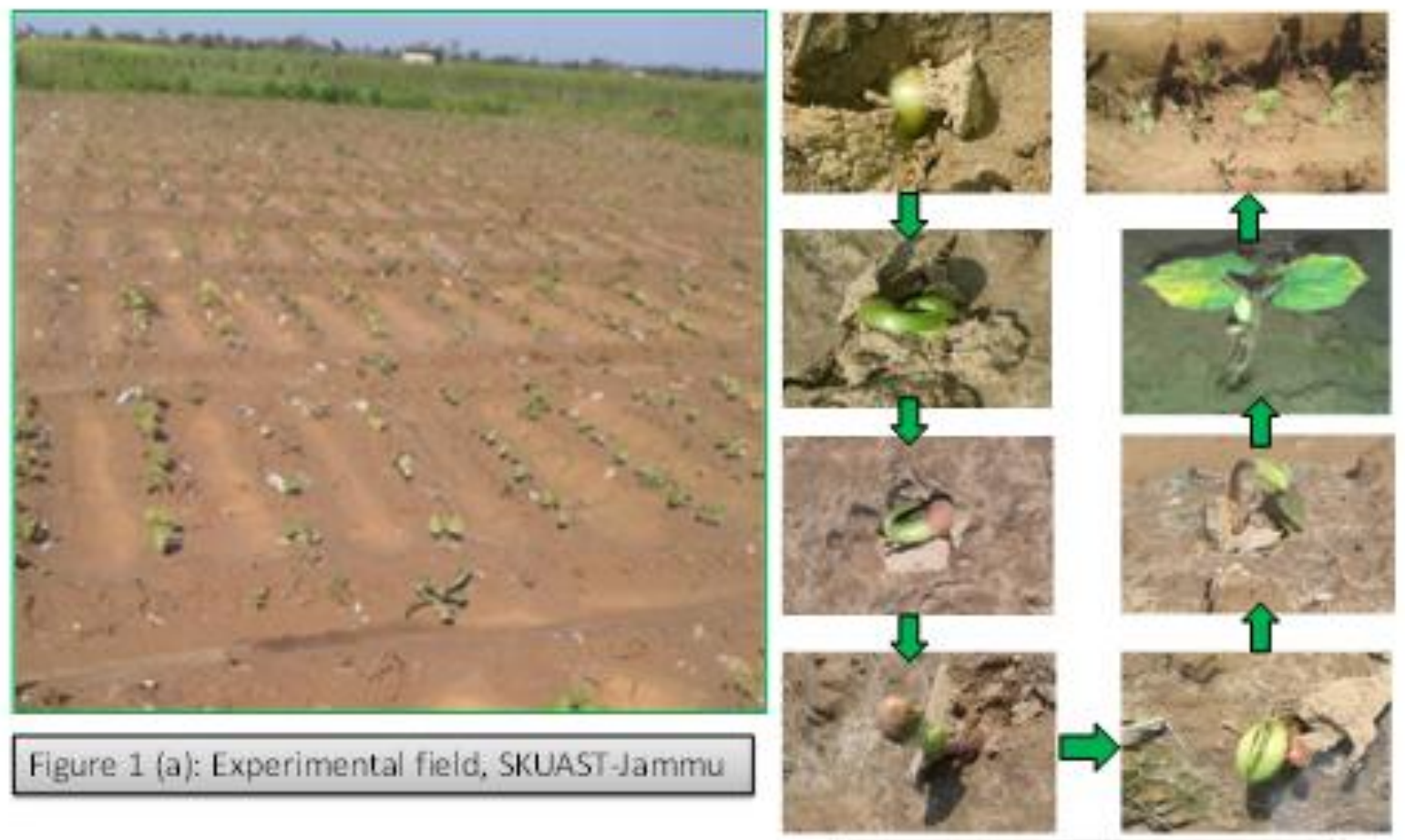

Figure 1 (b): Seed germination

Figure 1: Evaluation of divarea garmplasm hased on merphokgisal parametars

Further, we noticed considerably high values for genetic variability parameters such as heritability, genetic advance and genetic gain for 100 seed weight and seeds/plant traits (Table 3). However, genetic advance was found considerably low in case of days to bud initiation, days to flowering, days to fruiting, pod length and pod width. Heritability was high for the field influencing traits which is a desired characteristic that suggests direct selection of lines for improvement. Overall a substantial amount of divergence is observed in the collected common bean germplasm.

The presence of substantial amount of variability presents considerable scope for improvement of the studied characters. These results are in accordance with the findings of previous studies (Boros et al., 2014; Madakbasi et al., 2011) that recorded high variation for morphological traits in common bean germplasm. However, moderate morphological diversity was observed for tropical common bean germplasm comprised of 284 bean accessions, including 15 lines from Colombia, one line from Rwanda and 268 landraces (Okii et al., 2014). The genetic improvements utilizing various molecular breeding techniques are stable across beangrowing environments (Welch and Graham, 2004). Further, environment plays an important role in the accumulation of variation in geographically isolated individuals or genotypes present in different regions (Sarwat et al., 2008). Knowledge of the patterns of genetic variation can aid 
efficacious conservation of vulnerable species and provide information for sampling strategies for ex-situ and in-situ conservation (Torre et al., 2008). There is need for collection and acquisition of wild and cultivated species to conserve crop genetic diversity. Study of interspecies genetic variation facilitates the assessment of extinction risks such as inbreeding and evolutionary potential in a changing world. The knowledge of inheritance mechanism formulates appropriate breeding strategies for the improvement of a particular trait. In our study, minimum days to flowering and days to fruiting were taken by K12, VLR-125, N2, K13, KS10, MR1 and P2 which showed good potential in terms of yield as well. The data suggested that there is sufficient genetic variability to increase yield performance in common bean germplasm. The lines with desirable traits such as early maturity and high yield can be used for the purpose of enhancement of yield performance. These lines may be deployed in molecular breeding for the development of biparental populations and in back cross breeding programs aimed at enhancing yield in common bean.

\section{Summary and future prospects}

For development of varieties with extended adaptability and wide genetic base, it is must to have prior knowledge of variation in the existing germplasm. The study presented here will provide more possibilities for identification and selection of suitable genotypes which can be properly utilized for improvement of common bean. Diversity studies will provide a platform to better understand the crop structure for predicting cross combinations to get desirable F1 hybrids. Study of variability in terms of their traits and phylogenetic relationship forms the foundation of any breeding programme as it provides an opportunity to develop improved crops by more efficient sampling of genotypes and to exploit these varieties at commercial level. Thus, germplasm characterization of common bean ensures its efficient use in breeding programmes as new sources of variation will be utilized to identify and curtail yield limiting factors thereby enhancing the nutritional potential of the crop for alleviating hidden hunger with little recurring costs.

\section{Acknowledgement}

Financial support from DST for the INSPIRE Fellowship awarded to Nancy Gupta and SERB, DST, New Delhi for the project sanctioned to Dr. S.M. Zargar (Order No. SR/FT/LS-27/ 2011) is highly regarded.

\section{References}

Agriculture and Agri-Food Canada. 2009. Dry Bean Profile. http://www4.agr.gc.ca/ AAFCAAC/display-afficher.do?id=117 4576605314\&lang=eng Cited 25 March 2009.

Akibode, S. and Maredia, M. 2011. Global and regional trends in production, trade and consumption of food legume crops. SPIA Report, Department of Agricultural, Food and Resource Economics, Michigan State University, East Lansing, MI.

Allard, R.W. 1960. Principals of Plant Breeding. pp. 485. John willey and sons. Inc. New York.

Anonymous, 2015. http://www.cgiar.org/ourresearch/crop-factsheets/beans.

Bellucci, E., Bitocchi, E., Rau, D., Rodriguez, M., Biagetti, E., Giardini, A., Attene, G., Nanni, L. and Papa, R. 2014. Genomics of origin, domestication and evolution of Phaseolus vulgaris. In: Genomics of Plant Genetic Resources Tuberosa, R., Graner, A. and Frison, E. (Eds.) pp. 483-507.

Blumenthal, M., Busse, W.R., Goldberg, A., 
Grenwald, J., Hall, T., Riggins, C.W. and Rister, R.S. 1998. The Complete German Commission E Monographs Therapeutic Guide to Herbal Medicines. The American Botanical Council, Pub. Integrative Medicine Communications, Boston. Mass. USA. 157.

Boros, L., Wawer, A. and Borucka, K. 2014. Morphological, phenological and agronomical characterisation of variability among common bean (Phaseolus vulgaris L.) Local populations from the national centre for plant genetic resources: Polish genebank. Journal of Horticultural Research, 22 (2): 123-130.

Bressani, R. 1983. Research needs to upgrade the nutritional quality of common beans (Phaseolus vulgaris). Plant Foods for Human Nutrition Journal, 32: 101-110.

Burton, G.W. and DeVane, E.H. 1953. Estimating heritability in tall Fescue (Festuca arundiaceae) from replicated clonal material. Agronomy Journal, 45: 478-481.

CIAT report. 2001. http://webapp.ciat.cgiar. org/ciatinfocus/beans.htm

Cortes, A.J. 2013. On the origin of common bean (Phaseolus vulgaris L.). American Journal of Plant Sciences, 4: 19982000.

FAOSTAT. 2015. FAO Statistic. Food and agriculture organization of the United Nations (FAO). http://faostat3.fao.org /compare/E Accessed 28 January 2016.

Freytag, G.F. and Debouck, D.G. 2002. Taxonomy, distribution, and ecology of the genus Phaseolus (LeguminosaePapilionoideae) in North America, Mexico and Central America. Botanical Research Institute of Texas, Ft. Worth

Gepts, P., Aragao, F.J.L., de Barros, E., Blair, M.W., Brondani, R., Broughton, W., Galasso, I., Hernandez, G., Kami, J., Lariguet, P., McClean, P., Melotto, M.,
Miklas, P., Pauls, P., Pedrosa-Harand, A., Porch, T., Sanchez, F., Sparvoli, F. and $\mathrm{Yu}, \mathrm{K}$. 2008. Genomics of Phaseolus Beans, a major source of dietary protein and micronutrients in the tropics. Genomics of Tropical Crop Plants, 1: 113-143.

Johnson, H.W., Robinson, H.F. and Comstock, R.E. 1955. Estimation of genetic and environment variability in soybean. Agronomy Journal, 47: 314318.

Liebenberg, A. J. (Ed) 2000. Dry Bean Production. Department of agriculture directorate agricultural information service, Private Bag X144, Pretoria 0001 South Africa.

Madakbasi, S.Y. and Ergin, M. 2011. Morphological and phenological characterization of Turkish bean (Phaseolus vulgaris L.) genotypes and their present variation states. African Journal of Agricultural Research, 6 (28): 6155-6166.

Mamidi, S., Rossi, M., Annam, D., Moghaddam, S., Lee, R., Papa, R. and McClean, P. 2011. Investigation of the domestication of common bean (Phaseolus vulgaris) using multilocus sequence data. Functional Plant Biology, 38: 953-967.

Messina, M.L. 1999. Legumes and soybeans: overview of their nutritional profiles and health effects. The American Journal of Clinical Nutrition, 70: 439S$450 \mathrm{~S}$.

Okii, D., Tukamuhabwa, P., Odong, T., Namayanja, A., Mukabaranga, J., Paparu, P. and Gepts, P. 2014. Morphological diversity of tropical common bean germplasm. African Crop Science Journal, 22 (1): 59-67.

Palomino, V.R. 2012. Bayesian analysis of a linear mixed model to measure the impact of climate change on yield of common bean for the year 2030 
worldwide. Master's Thesis, University of Puerto Rico, Mayaguez, Puerto Rico.

Rafi, M.M. and Vastano, B.C. 2002. Novel polyphenol molecule isolated from licorice root (Glycrrhiza glabra) induces apoptosis, G2/M cell cycle arrest, and Bcl-2 phosphorylation in tumor cell lines. Journal of Agriculture and Food Chemistry, 50: 677-684.

Sarwat, M., Das, E.S. and Srivastava, E.P.S. 2008. Analysis of genetic diversity through AFLP, SMPL, ISSR and RAPD markers in Tribulus terrestris, a medicinal herb. Plant Cell Reports, 27: 519-528.

Schmutz, J., McClean, P.E., Mamidi, S., Wu, G.A., Cannon, S.B., Grimwood, J., Jenkins, J., Shu, S., Song, Q., Chavarro, C., Torres-Torres, M., Geffroy, V., Moghaddam, S.M., Gao, D., Abernathy, B., Barry, K., Blair, M., Brick, M.A., Chovatia, M., Gepts, P., Goodstein, D.M., Gonzales, M., Hellsten, U., Hyten, D.L., Jia, G., Kelly, J.D., Kudrna, D., Lee, R., Richard, M.M.S., Miklas, P.N., Osorno, J.M., Rodrigues, J., Thareau, V., Urrea, C.A., Wang, M., Yu, Y., Zhang, M., Wing, R.A., Cregan, P.B., Rokhsar, D.S. and Jackson, S.A. 2014. A reference genome for common bean and genome-wide analysis of dual domestications. Nature Genetics. 46 (7): $707-713$.
Singh, R.P. 1999. Common Bean Improvement in the Twenty-first Century. London, Kluwer Academic Publishers, pp xiii, $405 \mathrm{p}$

Singh, S.P. 2000. Bean research, production and utilization. In: Proc Idaho Bean Workshop, University of Idaho, Moscow, ID.

Torre, A., Lopez, S., Yglesias, E. and Cornelius, J.P. 2008. Genetic (AFLP) diversity of nine Cedrela odorata populations in Madre de Dios, southern Peruvian Amazon. Forest Ecology and Management, 255: 334-339.

Welch, R.M. and Graham, R.D. 2004. Breeding for micronutrients in staple food crops from a human nutrition perspective. Journal of Experimental Botany, 55: 353364.

WMO. 1992. The Global Climate System. Climate System Monitoring Dec 1988 May 1991. 73-74. WMO World Climate Data and Monitoring Programme, United Nations Environment Programme (UNEP), Nairobi, Kenya.

Wortmann, C.S., Brink, M. and Belay, G. 2006. Phaseolus vulgaris L. (Common bean). Record from PROTA4U. In: PROTA (Plant Resources of Tropical Africa / Ressources vegetales de

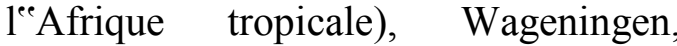
Netherlands. (Eds.) Brink, M. and Belay, G.

\section{How to cite this article:}

Nancy Gupta, Sajad Majeed Zargar, R.K. Salgotra, Manish Kumar Sharma, S.K. Gupta and Rai, G.K. 2019. Variability Estimates for Yield Determining Characters in Common Bean (Phaseolus vulgaris L.). Int.J.Curr.Microbiol.App.Sci. 8(08): 47-57. doi: https://doi.org/10.20546/ijcmas.2019.808.006 